\title{
Evidence for a population of high-redshift submillimeter galaxies from interferometric imaging
}

\author{
Alison B. Peck ${ }^{1}$ \\ Joint ALMA Office, Avda El Golf 40, piso 18, Santiago, Chile, 7550108 \\ E-mail: apeckealma.c1
}

Joshua D. Younger, Giovanni Fazio, Jiasheng Huang, Matthew Ashby, Mark Gurwell, Kamson Lai, Glen Petitpas, David Wilner

Harvard-Smithsonian CfA, 60 Garden St, Cambridge MA

E-mail: jyounger@cfa.harvard.edu

Min Su Yun, Grant Wilson, Kimberly Scott, Jason Austermann, Thushara Perera Astronomy Department, University of Massachusetts, Amherst, MA, 01003

\section{Daisuke lono, Kotaro Kohno}

Institute of Astronomy, University of Tokyo, 2-21-1, Osawa, Mitaka, Tokyo 181-0015

\section{Ryohei Kawabe}

NRO, Minamimaki, Minamisaku, Nagano 384-1805

\section{David Hughes, Itziar Aretxaga}

INAOE, Tonantzintla, Peubla, Mexico

\section{Tracy Webb}

Dept of Physics, McGill University, 3600 rue University, Montreal, Canada, H3A 2T8

\section{Alejo Martinez-Sansigre, Eva Schinnerer, Vernesa Smolcic} MPIA, Koeningstuhl-17, D-69117, Heidelberg, Germany

\section{Sungeun Kim}

Sejong University, 98 Kwangjin-gu, Kunja-dong, Seoul, 143-747, Korea

\section{James Lowenthal}

Smith College, Clark Science Center, Northampton, MA 01060

We have used the Submillimeter Array (SMA) to image a flux limited sample of seven submillimeter galaxies at $345 \mathrm{GHz}$ with 2" resolution. These galaxies were selected based on fluxes measured using the AzTEC camera on the JCMT at $1.1 \mathrm{~mm}$. All of the sources (two radiobright and five radio-dim) are unresolved, and we report positions accurate to $\sim 0.2 "$. All of these sources have multiple potential counterparts at other wavelengths within the original AzTEC beam. The astrometric accuracy offered by the SMA - which represents nearly an order of mag-

From planets to dark energy: the modern radio universe

University of Manchester, Manchester, UK

1-5 October, 2007

\footnotetext{
$1 \quad$ Speaker
} 
nitude improvement over single dish imaging - enables us to unambiguously identify counterparts at other wavelengths observed with similarly high angular resolution. Compared to the two radiobright sources in the sample, and those in previous studies, we find that the five radio-dim sources in the sample: 1) have systematically higher submm-to-radio flux ratios, 2) have lower IRAC 3.6$8.0 \mu \mathrm{m}$ fluxes, and 3) are not detected at $24 \mu \mathrm{m}$. These properties, combined with size constraints at $345 \mathrm{GHz}$, suggest that the radio-dim submm galaxies in our sample are representative of a population of very dusty starbursts, with physical scales for submm continuum emission similar to local ultraluminous infrared galaxies and an average redshift higher than radio-bright sources. Without the benefit of this positional accuracy, an incorrect counterpart would have been identified for at least two of these sources, resulting in erroneous interpretations of their physical properties.

Progress toward a thorough understanding of the physical processes driving submm galaxies (SMGs) has been hampered by two factors: their faintness at optical wavelengths, and the relatively poor $(\sim 10 ")$ angular resolution of the current generation of submm cameras. Although deep radio surveys, which showed a correlation between submm and radio continuum emission, localized SMGs to a few tenths of an arcsecond and allowed the first spectroscopic observations which showed that SMGs lie at high redshift $(2<z<3)$, only a fraction of these redshifts have been confirmed via CO or midIR spectroscopy. Furthermore, the rapid dimming of the radio continuum with $z$ means existing radio-confirmed SMG samples are relatively insensitive to systems at $z \sim 3$, and thus are biased. Recent studies have suggested that near- to mid-IR imaging using IRAC, in combination with $24 \mu \mathrm{m}$ observations using MIPS, on board the Spitzer Space Telescope, may offer an alternative to radio identification. However, this technique relies on either purely statistical arguments or broadband infrared color criteria, and also may be subject to biases.

Reliable counterpart identification represents potentially the most challenging obstacle to a more complete understanding of SMGs. Astrometry with the SMA highlights the unique power of this instrument; secure multi-wavelength counterparts for many of the targets could only be identified via interferometric imaging. We find that, while there is always - with the exception of the highly confused case of AzTEC2 - an IRAC counterpart coincident with the SMA position, there are often several $24 \mu \mathrm{m}$ sources within the AzTEC beam that are not associated with the submm emission. This is contrary to the prevailing wisdom, in which radio-dim SMGs, like their radio-bright counterparts, are associated with strong redshifted PAH emission features in the $24 \mu \mathrm{m}$ band. For all five of the radio-dim sources (AzTEC1-4 and AzTEC6) there are proximate $24 \mu \mathrm{m}$ sources that, if selected, would lead to misidentification of multiwavelength counterparts to the submm source. AzTEC5 has two potential radio counterparts within the AzTEC beam, both of which have strong $24 \mu \mathrm{m}$ emission. In this case as well, the SMA can unambiguously identify the correct counterpart. This study provides a preview of what will be much more easily achieved with ALMA in the near future, opening the door to followup studies with instruments at all wavelengths.

A full interpretation of the results shown here can be found in Younger et al (2007). 


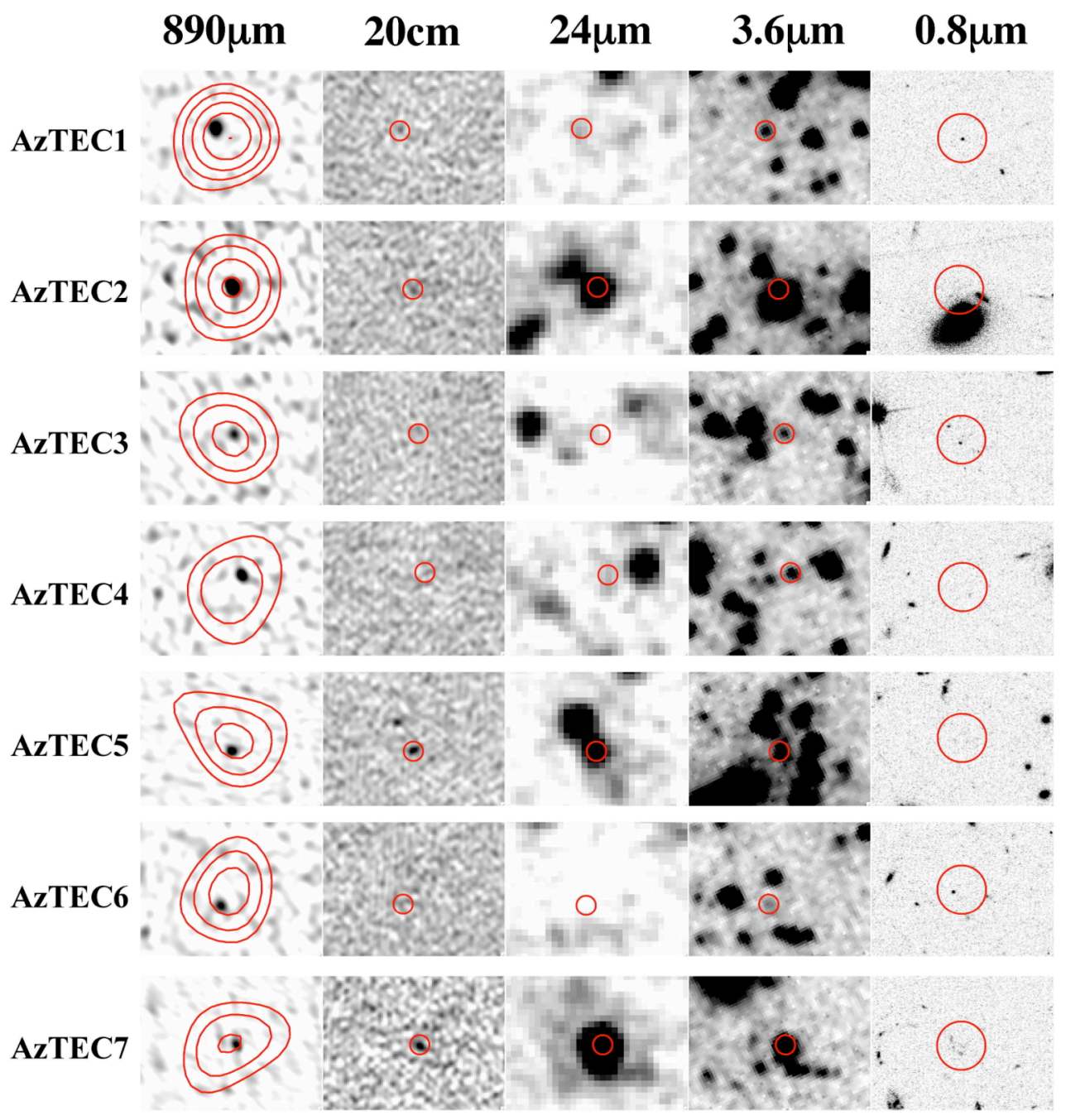

Fig 1. Postage stamp images for the SMA/AzTEC sources (top to bottom AzTEC1--7) for (left to right) SMA $(890 \mu \mathrm{m})$, VLA radio continuum $(20 \mathrm{~cm})$, MIPS Channel $1(24 \mu \mathrm{m})$, IRAC Channel $1(3.6 \mu \mathrm{m})$, and ACS ( $i$ band; $0.8 \mu \mathrm{m}$ ) imaging data. Overlaid in red on the SMA plots are contours at $3 \sigma, 4 \sigma, \ldots$ from AzTEC imaging data (Scott et al, 2007). The red circles in the remaining stamps have a radius 2", corresponding to twice the FWHM of the SMA beam, at the SMA position. Two sources (AzTEC 1 \& 7) have secure optical counterparts in the ACS images, while AzTEC 3 \& 6 have candidate optical counterparts that are a potential foreground object, and outside the SMA beam, respectively. Each stamp image is 37 " by 27 ", with the exception of the ACS stamps which are 15 " by 11 ".

\section{References}

[1] Scott, K. et al, 2007, in preparation

[2] Younger, J. et al 2007, arXiv:0708.1020 